\title{
Rated preference for musical compositions as it relates to complexity and exposure frequency
}

\author{
RONALD G. HEYDUK \\ Amherst College, Amherst, Massachusetts 01002
}

\begin{abstract}
Subjects heard four piano compositions that were constructed to represent differing degrees of complexity, as defined by their chordal and rhythmic properties and corroborated by subjects' complexity ratings. In line with the predictions of an optimal complexity model of musical preference, judged liking for the compositions was a unimodal function of their complexity. After each composition was rated for liking, one of the four compositions was presented and rated an additional 16 times. Also congruent with an optimal complexity model was the finding that the affective consequences of repeated exposure varied depending upon whether the repeatedly exposed composition was more or less complex than the subject's preferred complexity level. The latter finding suggests that repeated exposure effects are a function of both situational and individual factors.
\end{abstract}

Studies of preference for music and the hedonic consequences of musical repetition were common prior to the behaviorist revolution in experimental psychology. Verveer, Barry, and Bousfield (1933) report nine such experiments in an introduction to their own study of affective reactions to repeatedly exposed music. A significant study not included in their list was performed by Moore (1914), who collected experimental and historical data on the distribution of preferences for chords incorporating different harmonic relations and the nature of changes in preference with repeated exposure.

The majority of early experiments concerning musical preference used materials that had been previously composed rather than stimuli generated for research purposes. While the studies thus had a naturalistic quality, it was achieved at the expense of conceptual clarity. Stimulus attributes of the music that might have determined subjects' responses were not well defined (e.g., Gilliland \& Moore, 1924, merely compared reactions to "classical" and "popular" selections). Therefore, data collected with one set of materials were not easily reconciled with results of other experiments, and the possibilities for a general theory of musical preference were limited.

An integrative understanding of musical preference is aided by a conceptual framework such as that provided by Walker (1973), whose generic theory of exploratory and evaluative behavior incorporates ideas expressed by a number of others (Berlyne, 1960, 1971; Dember \& Earl, 1957; Eckblad, 1963; Fiske \& Maddi, 1961; Munsinger \& Kessen, 1964; Wundt, 1874). Walker subsumes the various stimulus attributes that have been proposed by the above authors to be motivationally relevant (i.e., novelty,

Based on a dissertation submitted in partial fulfillment of the requirements for a $\mathrm{PhD}$ degree at the University of Michigan. stimulus complexity, uncertainty, arousal properties) under a single label, psychological complexity, which is defined as a mutable characteristic of an individual's encounter with an object, not as a distal property of an object. Degree of preference for an event is postulated to be inversely related to the distance between the event's psychological complexity and an individual's optimal (preferred) psychological complexity level. Thus, an inverted $U$ function should occur when a subject expresses preferences for a range of musical events including some that are more complex and others that are less complex than is optimal. If the samples from a dimension of musical complexity are more limited in range, such that the event closest in psychological complexity to the person's optimal complexity level is the most or least complex of the set, then monotonic increasing or monotonic decreasing functions relating preference to complexity are the respective expectations.

By employing the additional assumption that experience with an event reduces its psychological complexity, predictions may also be made about the nature of preference changes with continued exposure to a musical selection. If repetition of a supraoptimal composition makes it less complex for the organism, its distance from the fixed point of maximum preference should decrease. Correspondingly, attractiveness should increase until continued simplification brings the event to the optimal complexity level; thereafter, further exposures to the same piece of music should result in a progressive decline in liking. ${ }^{1}$ This suggests that Zajonc's (1968) theory of repeated exposure effects is a special case of the optimal complexity model. Zajonc presents evidence that repetition of a stimulus produces an increase in its attractiveness. The current model proposes that both increases and decreases in liking may occur as a 

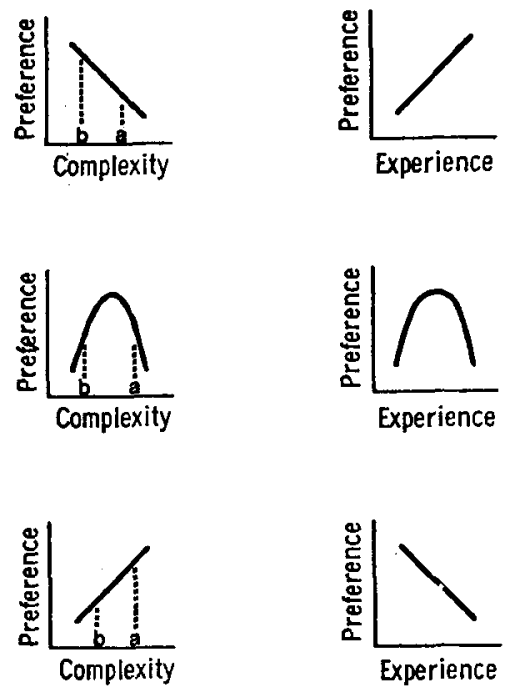

Figure 1. Relationships between preference and complexity and between preference and experience supporting an optimal complexity model. In each instance, the right-hand curve shows the trend in preference for a particular event as a function of experience that would be expected if, as a result of the experience, the event decreased in psychological complexity from Point a to Point $b$, as indicated on the adjacent left-hand curve.

function of exposure, depending upon whether the repeated event is more or less psychologically complex than the individual's optimal level. This analysis is congruent with casual observations that a person's hedonic reactions to repetition vary for different musical compositions, as do the reactions of different persons to the same piece of music.

In summary, since as a consequence of repetition a given event is expected to undergo simplification (i.e., become progressively less complex from the perspective of the organism), a prediction that event preference should show a single peak over a range of exposure frequencies is deducible from the prediction that preference is unimodally related to psychological complexity. Figure 1 illustrates the functional relationships that are congruent with these two major predictions, and demonstrates that the expected functions relating experience to preference may be derived from the functions relating complexity to preference.

Optimal stimulation models of aesthetic preference have been supported by a number of recent investigations which Berlyne (1971) labels collectively "the new experimental aesthetics." Several limitations common to the studies he cites prompted the present research.

(1) Lacking is an explicit test of the hypothesis that repeated exposure effects are a joint function of situational and individual factors. For example, in studies using visual materials, Berlyne (1970) and Saegert and Jellison (1970) supported the situational predictions of an optimal stimulation model: simple stimuli became less pleasant with repetition, while complex stimuli became more pleasant. However, the procedures used in these studies did not permit the location of an optimal preference point for each subject, making it impossible to test the stronger prediction that the consequences of repetition depend upon the complexity of an event as it relates to the individual's optimal level. The current paradigm allows such a test.

(2) Most of the relevant experiments have used visual stimuli (see Berlyne, 1971, and Walker, 1970, for summaries), and with few exceptions the auditory studies (see Berlyne, 1971, chap. 13, for summary) have employed stimulus materials that were clearly defined but did not combine the chordal, sequential, instrumental, and thematic characteristics of genuine aesthetic products adapted for experimental use. For example, in studying the effects of various stimulus parameters on preference, Vitz $(1964,1966,1972)$ used mechanically generated single tones and tone sequences, while Skaife (1967) had sequences of notes played on an electronic instrument and Berlyne, McDonnell, Nicki, and Parham (1967) presented tone combinations (chords) but not in sequence. The original piano compositions created for the present experiment were intended to engage listeners in a more natural music appreciation task without sacrificing a clear operational definition of complexity.

\section{METHOD}

\section{Subjects}

Subjects were 120 University of Michigan undergraduates. Forty males and 40 females fulfilled a part of their introductory psychology course requirement by serving without pay for a single 30 -min session. Another 20 male and 20 female students were paid for equivalent participation.

\section{Materials and Apparatus}

Stimulus materials consisted of four original musical compositions having a number of common features. Each was a piano solo lasting $30 \mathrm{sec}$ and having three parts: a $10-\mathrm{sec}$ original statement consisting of four bars with seven chord changes and no melody; a 15-sec middle section of six bars with five chord changes and some melody in the right hand; and a 5-sec resolution with three chord changes occurring over an interval of two bars. Tempo, meter, and tonal range were also the same for each composition.

Complexity differences were achieved by covarying two aspects of the chord structure and the amount and kind of syncopation. Composition $\mathrm{A}$, the least complex musical piece on an a priori basis, had only 2 different chords, both of which were common major triads. Composition $B$ had 4 different chords, 1 of which $(25 \%)$ was not a major triad. Composition $C$ used 8 different chords, 4 of them $(50 \%)$ other than major. Composition D, intended to be the most complex of the set, had 12 different chords, 8 of these $(75 \%)$ with a minor, seventh, or quartal structure.

Syncopation was varied as follows: A had chords played with both hands on measure beats only (no syncopation); B was played with a common syncopation in both hands; $C$ had syncopation in the left hand and none in the right hand; and D had different syncopations in the two hands. 
Moderato: $J=132$
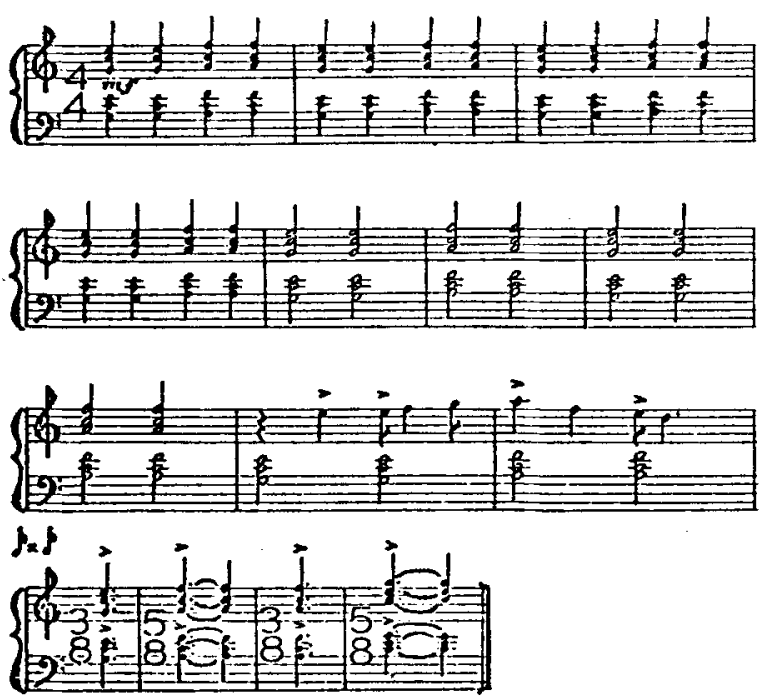

Moderato: $J=132$

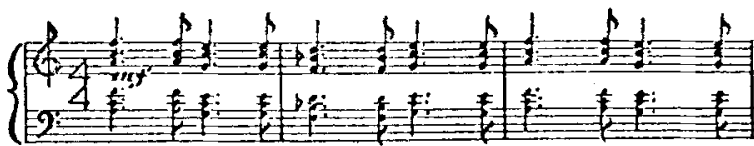
$\beta_{2} \boldsymbol{b}$
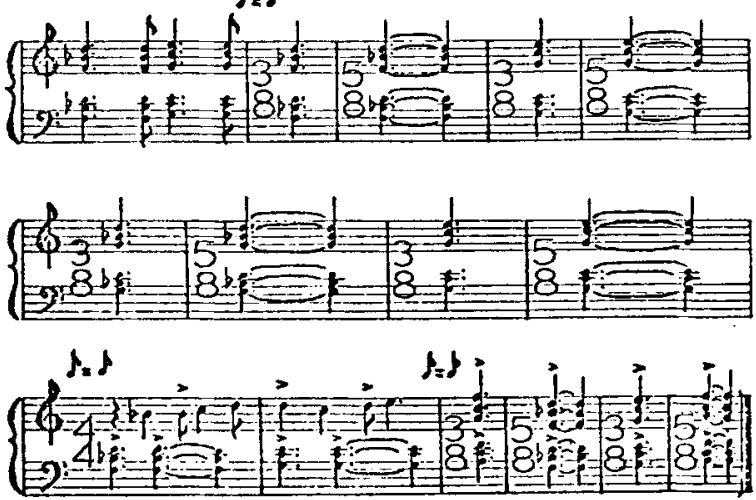

compostton C

compositon d́

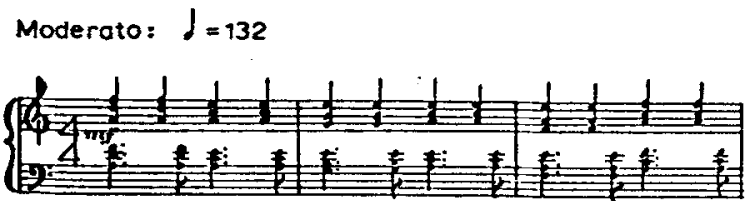

Moderato: $J=132$
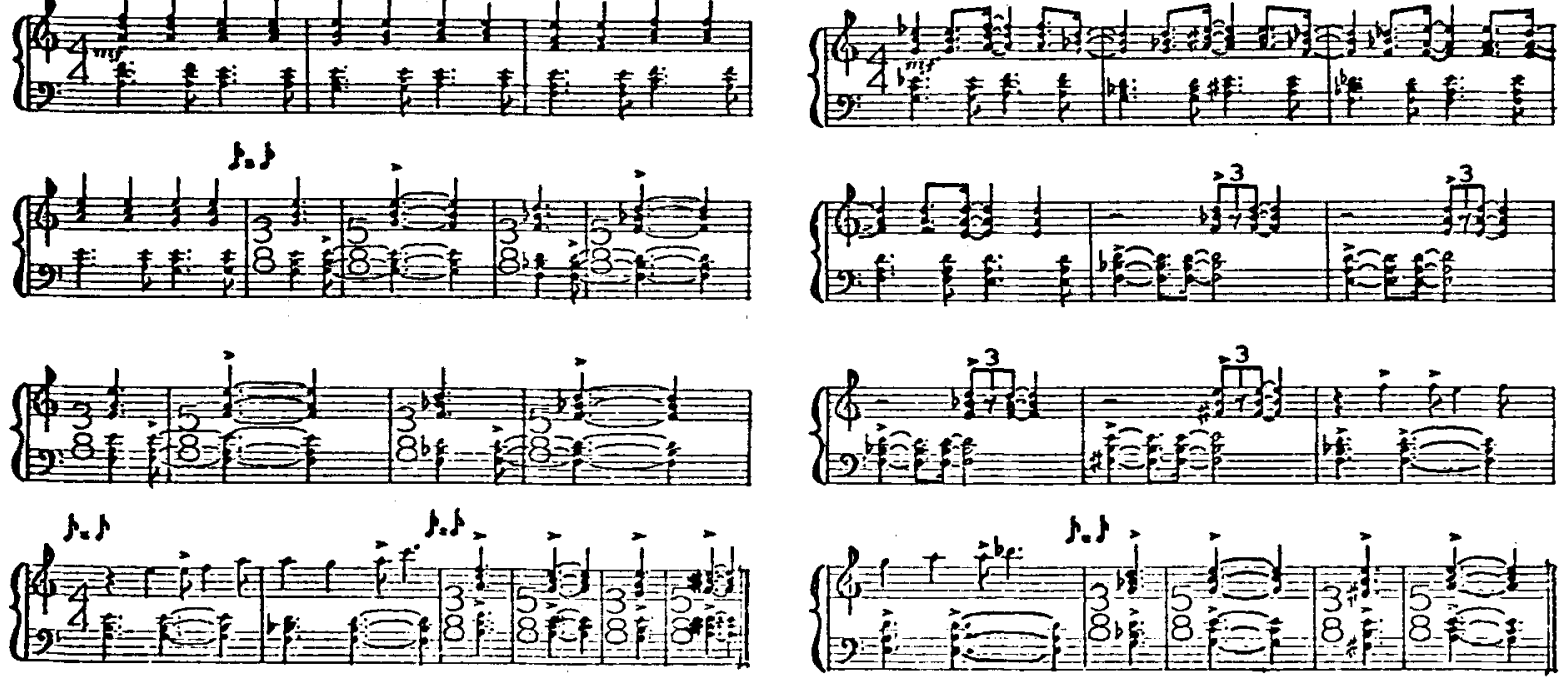

Figure 2. Sheet music for piano compositions used as musical stimuli.

The net effect was the creation of four thematically similar musical compositions that could be ordered on a simplicitycomplexity dimension on the basis of the redundancy and presumed familiarity of chords and syncopation. These characteristics were selected to define psychological complexity because of the suggested importance of disconfirming an organism's expectations (transmitting more information) in establishing greater complexity (Dember \& Earl, 1957; Vitz, 1964). Figure 2 contains the complete sheet music for the four compositions.

A Wollensak two-channel, reel-to-reel tape recorder operated at 


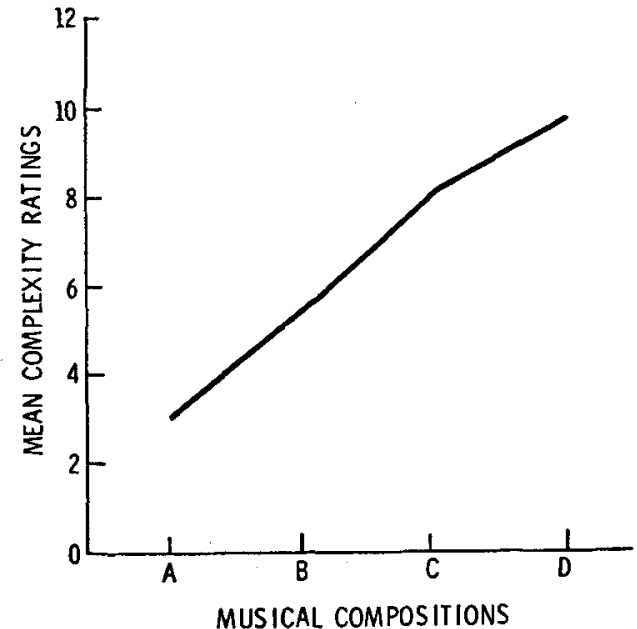

Figure 3. Mean rated complexity of musical compositions with the four compositions ordered according to construction procedures.

$71 / 2$ i.p.s. speed was the recording and presentation device. The compositions were recorded by the experimenter in a soundproofed room equipped with a Hamilton upright piano.

\section{Procedure}

Subjects were run in groups of 25 or less. In the initial phase of the experiment, the subjects were instructed to rate each composition for "how much you like it" and "how complex it sounds to you." Separate rating scales for liking and complexity appeared on a single rating sheet. The scales ranged from 1 to 13 , with qualitative judgment markers provided for every other number as follows: 13-"Like a lot" ("Extremely complex" on the complexity scale); 11-"Like" ("Complex"); 9-"Like a little" ("Somewhat complex"); 7-"Neutral"; 5-"Dislike a little" ("Somewhat simple"); 3-"Dislike" ("Simple"); 1-"Dislike a lot" ("Extremely simple"). Included on the rating sheet was the additional instruction to judge each composition relative to the others rather than compare each to an absolute standard.

After the instructions had been clarified, the experimenter played the tape with the four recorded compositions in one of four predetermined random orders (different stimulus orders were used for different subjects as a control for possible sequence-related effects). The interval between the playing of each piece was approximately $15 \mathrm{sec}$. During the second playing of the stimulus set, which followed the same order as the first, the subjects used the interstimulus interval for recording their numerical ratings, first for liking and then for complexity, on the rating sheet. In cases where any subjects had not yet rated the previous piece within $15 \mathrm{sec}$, extra time was allotted before the next piece was played.

Following the initial ratings of all four compositions, the subjects were informed that they would hear one of the four just-rated selections repeatedly and were asked to rate it for liking after each repetition. For this segment of the experiment, the 120 subjects were assigned to four groups as follows: 40 subjects (randomly selected) received 16 exposures to Stimulus D, 20 received 16 exposures to Stimulus $\mathrm{C}$, another 20 had a like number of exposures to $\mathrm{B}$, and 40 subjects listened to A 16 times. The initial ratings of the selected composition were recorded on the new rating sheet and were thus available to the subjects for reference.

After instructions, the subjects rated the single selected piece during the 15 interstimulus intervals of $15 \mathrm{sec}$ each, and again after the last playing. Thus, subjects listened to three of the four compositions twice each and the fourth 18 times, with ratings required following the second and all subsequent exposures.

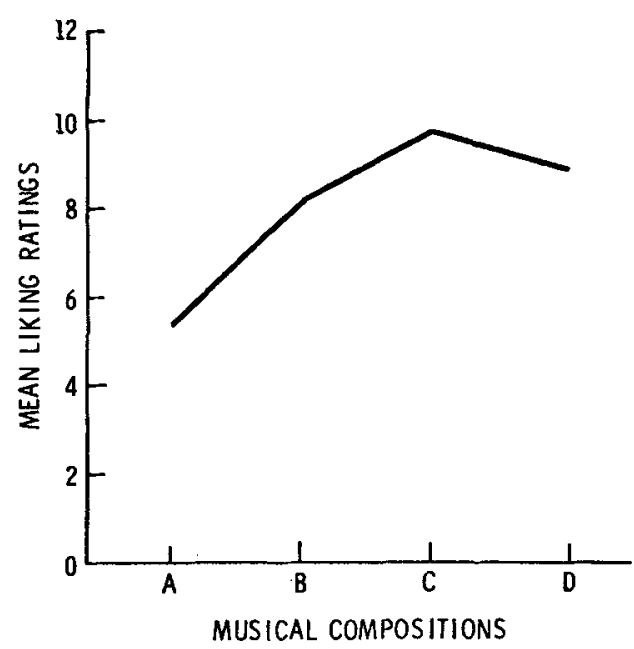

Figure 4. Mean rated liking of musical compositions on the initial rating occasion.

Completion of the entire experiment required approximately $25 \mathrm{~min}$.

\section{RESULTS AND DISCUSSION}

\section{Ratings of Complexity}

One basis for ordering the four stimuli in terms of psychological complexity derives from the stimulus construction procedure: chord structure and syncopation were manipulated such that the compositions may be ordered $\mathrm{D}>\mathrm{C}>\mathrm{B}>\mathrm{A}$. Another possible index of psychological complexity derives from the subjects' mean ratings of complexity. Figure 3 shows the relationship between complexity as defined by the stimulus construction procedure and mean rated complexity for the 120 subjects (equal spacing of stimuli on the abscissa is not intended to imply equal psychological distances). The monotonic increasing relationship means that the subjects' consensus opinion about complexity also yields the stimulus ordering $\mathrm{D}>\mathrm{C}>\mathrm{B}>\mathrm{A}$. This congruence between the a priori and rating-derived measures supports the assumption that the four compositions cover a discriminable range of psychological complexity.

\section{Ratings of Liking: Initial}

The group preference function (mean rated liking

Table 1

Rated Liking for Complexity: Individual Protocol Analysis

\begin{tabular}{|c|c|c|c|c|}
\hline \multicolumn{3}{|c|}{ Supporting Protocols } & \multirow[b]{2}{*}{ df } & \multirow[b]{2}{*}{$x^{2}$} \\
\hline $\begin{array}{l}\text { Observed } \\
\text { Number }\end{array}$ & $\begin{array}{c}\text { Expected } \\
\text { Proportion }\end{array}$ & $\begin{array}{c}\text { Expected } \\
\text { Number }\end{array}$ & & \\
\hline 85 & .356 & 42.7 & 1 & $64.0^{*}$ \\
\hline
\end{tabular}


on the first rating occasion vs. complexity) appears in Figure 4 as the expected inverted $U$ : the point of model preference is at Composition $C$.

A more demanding test of the optimal complexity model results when each subject's liking ratings, rather than merely contributing to a group curve, are inspected individually for unimodality with respect to complexity. Table 1 summarizes the analysis of rated liking for individuals. It reveals that, for 85 subjects, liking was a perfect single-peaked (inverted $\mathrm{U}$, monotonic increasing, or monotonic decreasing) function of complexity. Thus, the preference orders of 85 of the 120 subjects were completely congruent with an optimal complexity model, significantly more than would be expected if chance alone were operating $\left[\chi^{2}(1)=64.0, \mathrm{p}<.001\right] .^{2}$

When considered together, group and individual data analyses suggest that the affective response of a subject to a musical composition was influenced by its proximity to the subject's preferred complexity level. ${ }^{3}$

\section{Ratings of Liking: Repeated Exposure to a Single Composition}

The current experimental paradigm was well suited for testing the hypothesis that the affective consequences of musical repetition depend on a relationship between situational and individual factors. Prior to the repeated exposure portion of the experiment, the necessary information was available for classifying each subject according to the sort of function relating preference to exposure frequency that should have resulted for him. A subject's original liking ratings of the four compositions determined the location of his optimal complexity level (e.g., if modal liking was exhibited for $C$, the optimal complexity level was closer to $C$ than to $B$ or $D$ ). If the composition to be repeatedly presented was more complex than the individual's optimum, the expected simplification resulting from further exposure should have brought it closer to the preferred point, and liking for it should have increased, at least until the optimum was reached. Thus, a monotonic increasing (or inverted $\mathrm{U}$ ) function relating preference and exposure frequency was predicted. If, on the other hand, the to-be-presented musical selection was already less complex than the optimum when repetition of it began, then only a subsequent monotonic decrease in liking was regarded as supporting the optimal complexity model.

Using these criteria, subjects were assigned on an a priori basis to one of two categories, one for which the prediction was that subsequent exposures to the selected composition would lead to a monotonic increasing or inverted $U$ relation between liking and exposures, and the other category for which monotonic decreasing functions were expected. ${ }^{4}$ Then each subject's average liking ratings for Exposure
Table 2

Relationship Between Predicted and Observed Curve Types for Subjects Whose Rated Liking Was a Unimodal Function of Exposure Frequency

\begin{tabular}{lccc} 
& \multicolumn{3}{c}{ Predicted } \\
\cline { 2 - 4 } & $\begin{array}{c}\text { Monotonic } \\
\text { Increasing } \\
\text { or Inver- } \\
\text { ted U }\end{array}$ & $\begin{array}{c}\text { Mono- } \\
\text { tonic } \\
\text { Decreas- } \\
\text { ing }\end{array}$ & Total \\
\hline Observed & 15 & 22 & 37 \\
Monotonic Increasing & 11 & 40 & 51 \\
Monotonic Decreasing & 26 & 62 & 88 \\
Total & & & \\
\hline
\end{tabular}

Trials 1-5, 6-10, and 11-15 were computed and the functional relation between exposure trial blocks and liking examined.

Twenty subjects showed no changes in rated liking across the three exposure blocks, and their protocols were not considered further. Of the remaining 100 subjects, another 12 exhibited functions not fitting either category (i.e., bimodal functions) and were also eliminated from further consideration. The 88 remaining unimodal functions were subsequently classified on the basis of whether maximum preference was exhibited during the first trial block (monotonic decreasing function) or during the second or third blocks (inverted-U or monotonic increasing functions). By comparing the a priori and data-based classification of subjects, the success of the optimal complexity model in predicting the relationship between preference and exposures could then be determined. A 2 by 2 contingency table (Table 2) reveals that predicted and observed curve types corresponded for 55 subjects. Fifteen monotonic increasing or inverted $U$ functions and 40 monotonic decreasing functions were predicted accurately: the other 33 predictions proved inaccurate. By way of comparison, only 21 subjects exhibited the consistent upward trend in liking that conforms to Zajonc's (1968) view of the consequences of repetition.

More relevant for determining the value of an optimal complexity approach to repeated exposure is the relationship between the a priori and data-based classifications of the subjects. A lack of association between prediction and observation would indicate that knowledge of the composition's complexity and the individual's optimum did not aid prediction of the consequences of further repetition. Table 2 suggests, however, that an association does exist: the distributions of observed curve types in the two prediction categories are different, and the difference is in the expected direction. A chi-square test for independence reveals that the positive association between prediction and observation is significant $\left[\chi^{2}(1)=2.85, \mathrm{p}<.05\right.$, one-tailed test $]$. We may conclude that the information about an individual's 
optimal level gained from initial liking ratings was useful for predicting subsequent hedonic responses to repetition of an event with specified complexity.

In defending the hypothesis that repeated exposure to stimuli produces steady increases in affect against evidence that decreases in liking are often observed, Zajonc (1974) argues that such decreases are merely a temporary satiation phenomenon resulting from massed exposures to the stimulus material. The present data is at least consistent with the idea that for some subjects satiation may have led to decreased liking when increased liking might have occurred if exposures had been less concentrated. However, a simple satiation hypothesis cannot explain the fact that although all subjects received massed exposures to a composition, steady drops in liking were more common among subjects whose preferred level of complexity was greater than the presented composition.

\section{Concluding Comments}

In summary, several aspects of subjects' rated liking for four original piano compositions agreed with predictions of a theory of psychological complexity and preference proposed by Walker (1973). Mean rated liking was unimodally related to the complexity of the compositions. Single-peaked functions were also the rule for individual subjects: once a subject's preferred composition was known, the ordinal aspects of liking for the other three compositions were predictable with a frequency significantly greater than chance. In addition, predictions about the nature of liking changes as a function of repeated exposure to a single composition were successful at a better-than-chance rate. Above-optimum pieces were liked more with experience and below-optimum pieces were liked less with experience.

The optimal complexity model that generated predictions for this study proposes that behavior is a product of a relationship between a situational factor (psychological complexity) and a parameter of the individual (optimal complexity level). By combining in a single "behavior equation" constructs which historically have been the province of different schools of psychology, the model meets the specifications for the ideal model set forth by Lewin (1946) and again by Cronbach (1957). Whether the strategy of combining information about the individual and the situation in order to improve prediction is as valuable in practice as it is conceptually appealing is an important research question. Analysis of the repeated exposure data suggested that the strategy may be useful, but in the case of the initial liking data a critical test was not possible. Since no measure of an individual's optimal complexity level existed before the ratings were collected, general predictions of unimodal preference with respect to complexity were all that could be made. Future research on preference for complexity should be directed toward developing a ratingindependent measure of the optimal complexity level so that for each subject a specific prediction about the preference-complexity relationship will be possible.

\section{REFERENCES}

Berlyne, D. E. Conflict, arousal, and curiosity. New York: McGraw-Hill, 1960.

Berlyne, D. E. Novelty, complexity, and hedonic value. Perception \& Psychophysics, 1970, 8, 279-286.

Berlyne, D. E. Aesthetics and psychobiology. New York: Appleton, 1971.

Berlyne, D. E., McDonnell, P., Nicki, R. M., \& Parham, L. C. C. Effects of auditory pitch and complexity on EEG desynchronization and on verbally expressed judgments. Canadian Journal of Psychology, 1967, 21, 346-367.

Cronbach, L. J. The two disciplines of scientific psychology. American Psychologist, 1957, 12, 671-684.

Dember, W. N., \& EARL, R. W. Analysis of exploratory, manipulative, and curiosity behavior. Psychological Review, 1957, 64, 91-96.

ECKBLAD, G. The attractiveness of uncertainty. Scandinavian Journal of Psychology, 1963, 4, 1-13.

Fiske, D. W., \& MADDI, S. R. A conceptual framework. In D. W. Fiske and S. R. Maddi (Eds.), The functions of varied experience. Homewood, Ill: Dorsey, 1961.

Gilliland, A. R., \& Moore, H. T. The immediate and long-time effects of classical and popular phonograph selections. Joumal of Applied Psychology, 1924, 8, 309-323.

HeyDuk, R. G. Static and dynamic aspects of rated and exploratory preference for musical complexity. Unpublished doctoral dissertation, University of Michigan, 1972.

Lewin, K. Behavior and development as a function of the total situation. In L. Carmichael (Ed.), Manual of child psychology. New York: Wiley, 1946.

Miller, G. A. The magic number seven, plus or minus two: Some limits on our capacity for processing information. Psychological Review. 1956, 63, 81.97.

Moore, H. T. The genetic aspects of consonance and dissonance. Psychological Monographs, 1914, 17(2, Whole No. 73).

Munsinger, H., \& Kessen, W. Uncertainty, structure, and preference. Psychological Monographs, 1964, 78(9, Whole No. 586).

NeISSER, U. Cognitive psychology. New York: Appleton-CenturyCrofts, 1967.

SaEgert, S. C., \& Jellison, J. M. Effect of initial level of response competition and frequency of exposure on liking and exploratory behavior. Joumal of Personality and Social Psychology, 1970, 16, 553-558.

SKAIFE, A. M. The role of complexity and deviation in changing taste. Unpublished doctoral dissertation, University of Oregon, 1967.

Verveer, E, M., Barry, H., \& Bousfield, W. A. Change in affectivity with repetition. American Journal of Psychology, 1933, 45, 130-134.

VITZ, P. C. Preference for rates of information presented by sequences of tones. Journal of Experimental Psychology, 1964, 68, $176-183$

VITZ, P. C. Affect as a function of stimulus variation. Journal of Experimental Psychology, 1966, 71, 74-79.

$V_{\text {ITZ, P. C. Preference for tones as a function of frequency }}$ (hertz) and intensity (decibels). Perception \& Psychophysics, 1972, 11, 84-88.

WALKER, E. L. Psychological complexity as a basis for a theory of motivation and choice. In D. Levine (Ed.), Nebraska 
symposium on motivation 1964. Lincoln: University of Nebraska Press, 1964.

WALKER, E. L. Complexity and preference in animals and men. Annals of the New York Academy of Sciences, 1970, 169, 619-652.

WALKER, E. L. Psychological complexity and preference: A hedgehog theory of behavior. In D. E. Berlyne and K. B. Madsen (Eds.), Pleasure, reward, preference. New York: Academic Press, 1973.

Wund, W. Grundzuge der physiologischen Psychologie. Leipzig: Englemann, 1874.

ZAJoNC, R. B. Attitudinal effects of mere exposure. Journal of Personality and Social Psychology, 1968, 9 (Monograph Supplement 2, Part 2).

ZaJoNC, R. B. Effect of extreme exposure frequencies on different affective ratings of stimuli. Perception and Motor Skills. 1974, 38, 667-678.

\section{NOTES}

1. An alternate interpretation of the effects of exposure to complexity is that the individual's optimal level of complexity increases while complexity of the stimulus remains constant. Although, in terms of major predictions, this interpretation is functionally equivalent to the "decreasing event complexity, fixed optimum" view, the latter is preferable in two respects. For one, it is congruent with currently popular conceptions of the organism as one whose experience of the environment is constructive and mutable (Neisser, 1967) and who has a relatively fixed capacity to process information (Miller, 1956). In addition, there is evidence that the preferred interpretation is more consistent with observed sequences of choices among events differing in complexity: see Walker (1964) for a discussion.

2. If Compositions $A$ or $D$ are rated as most preferred, there are 12 ways of ordering preference for the remaining three compositions, 2 of which (1/6) produce preference orderings that are unimodal functions of complexity. If Compositions $\mathrm{B}$ or $\mathrm{C}$ are given the highest liking rating, 6 of the possible 12 orderings of the remaining three compositions $(1 / 2)$ are unimodal. Thus, $1 / 6$, or 8.7 , of the 52 subjects who liked $A$ or $D$ best and $1 / 2$, or 34 , of the remaining 68 subjects (a total of 42.7 of the 120 ) would have been expected to exhibit unimodal preference orderings through the workings of chance alone.

3. Additional analyses of the same data (e.g., by subgroups, by compositions) also supported an optimal complexity interpretation (see Heyduk, 1972, pp. 24-36).

4. When the modal preference point for a subject coincided with the composition that had been randomly assigned to him for repeated exposure (indicating that the optimal complexity level was closer to this composition than to any other), a prediction of a monotonic decrease in liking with exposure was made. Although, under these conditions, the appearance of increases in liking over blocks of exposures would not controvert the model in the strictest sense, the conservative strategy of rejecting such increases as support for the modęl was decided upon because it was assumed that any initial increases in liking would be accomplished by the end of the first block of exposure trials, after which only liking decreases should occur.

(Received for publication July 1, 1974; revision accepted September 11, 1974.) 